\title{
POINTWISE CHAIN RECURRENT MAPS OF THE SPACE $Y$
}

\author{
Wenjing Guo, FanPing Zeng and QIYING Hu
}

Let $Y=\left\{z \in C: z^{3} \in[0,1]\right\}$ (equipped with subspace topology of the complex space $C)$ and let $f: Y \rightarrow Y$ be a continuous map. We show that if $f$ is pointwise chain recurrent (that is, every point of $Y$ is chain recurrent under $f$ ), then either $f^{12}$ is the identity map or $f^{12}$ is turbulent. This result is a generalisation to $Y$ of a result of Block and Coven for pointwise chain recurrent maps of the interval.

\section{INTRODUCTION}

In this paper we characterise the dynamics of maps of the space $Y=\{z$ $\left.\in C: z^{3} \in[0,1]\right\}$ equipped with the subspace topology for with every point is chain recurrent. We prove the following.

MAIN THEOREM. Let $f$ be a continuous map of $Y$ to itself. If $f$ is pointwise chain recurrent, then either $f^{12}$ is the identity map or $f^{12}$ is turbulent.

Block and Coven (see [4]) proved that a pointwise chain recurrent map $h$ of the interval must satisfy that either $h^{2}$ is the identity map or $h^{2}$ is turbulent. So our theorem extends this result to maps of the space $Y$.

Firstly some notation and definitions are established. Let $(X, d)$ be a compact metric space and $g: X \rightarrow X$ be a continuous map. If $g^{n}(x)=x \neq g^{k}(x), k=1,2, \ldots, n-1$, for some $x \in X$ and some positive integer $n$, then the point $x$ is called a periodic point of period $n$, where $g^{0}=i d, g^{i}=g \circ\left(g^{i-1}\right)(i \geqslant 1)$. In particular, if $g(x)=x$, then $x$ is called a fixed point of $g$. Denoted by $P(g)$ and $F(g)$ the set of periodic points and fixed points set of $g$ respectively. For $x, y \in X$ and $\varepsilon>0$, an $\varepsilon$-chain from $x$ to $y$ is a finite sequence $x=x_{0}, x_{1}, \ldots, x_{n-1}, x_{n}=y$ with $d\left(g\left(x_{i}\right), x_{i+1}\right)<\varepsilon$ for $0 \leqslant i \leqslant n-1$. We say $x$ is chain recurrent under $g$, if for each $\varepsilon>0$, there is an $\varepsilon$-chain from $x$ to $x$. The map $g$ is said to be pointwise chain recurrent, if every point of $\mathrm{X}$ is chain recurrent under $g$. The following facts about chain recurrent are standard observations:

(a) If $g$ is pointwise chain recurrent, then $g$ maps $X$ onto $X$.

(b) $g$ is pointwise chain recurrent if and only if $g^{n}$ is pointwise chain recurrent for every $n>0$.

Received 8th May, 2002

Project supported by NNSF of China (19961001).

Copyright Clearance Centre, Inc. Serial-fee code: 0004-9727/03 \$A2.00+0.00. 
(c) [5, Theorem A] If $X$ is connected and $g: X \rightarrow X$ is pointwise chain recurrent, then there is no nonempty open set $U \neq X$ such that $g(\bar{U}) \subseteq U$.

Being chain recurrent is an important dynamical property of a stystem and has been studied intensively in recent years. For more details see $[\mathbf{1}, \mathbf{3}, \mathbf{5}, \mathbf{6}, \mathbf{9}]$.

The space $Y$ is obviously a tree (see [7]) in which there are exactly three ends, denoted by $e_{1}, e_{2}$, and $e_{3}$, and exactly one vertex, denoted by $o$. For $a, b \in Y$, We shall use $[a, b]$, called a closed subinterval of $Y$, to denote the smallest closed connected subset containing $a$ and $b$. We define $(a, b)=[a, b] \backslash\{a, b\}$ and we can similarly define $(a, b]$ and $[a, b)$. For a subset $A$ of $Y$, we use $\operatorname{int}(A), \bar{A}$ and $\partial A$ to denote the interior, the closure and the boundary of $A$, respectively.

A map $g: Y \rightarrow Y$ is called turbulent if there are closed subintervals $J$ and $K$ with disjoint interiors such that $g(J) \cap g(K) \supseteq J \cup K$. Clearly, if $f$ is turbulent then $f^{n}$ is turbulent for any $n \geqslant 2$.

From the above definition of turbulence and the proof of $[8$, Theorem 1$]$, the following result is clear.

THEOREM 1.1. Let $f$ be a continuous map of space $Y$. If $f$ is turbulent, then $f$ has more than one fixed point.

Let $e \in\left\{e_{1}, e_{2}, e_{3}\right\}$. A partial order $<_{e}$ on $Y$ defined as follows, which will be useful in dealing with continuous maps of the space $Y$. For $x, y \in Y, x<_{e} y$ if $x \in[y, e]$ and $x \neq y$.

Throughout this paper, $f$ denotes a pointwise chain recurrent map of $Y$ into itself. This paper is organised as follows. In Section 2 and Section 3, the pointwise chain recurrent maps of $Y$ with more than one fixed point are characterised, where the fixed points set is disconnected in Section 2 and connected in Section 3. In Section 4, the pointwise chain recurrent maps of $Y$ with exactly one fixed point are discussed.

EXAmples. Clearly, $Y=I \cup\left\{x e^{(2 / 3) \pi i} \mid x \in I\right\} \cup\left\{x e^{(4 / 3) \pi i} \mid x \in I\right\}$, where $I=[0,1]$.

(1) $f: Y \rightarrow Y, f(x)=x e^{(2 / 3) \pi i}, f\left(x e^{(2 / 3) \pi i}\right)=x$ and $f\left(x e^{(4 / 3) \pi i}\right)=x e^{(4 / 3) \pi i}$ for any $x \in[0,1]$. Then $f$ is pointwise chain recurrent such that $f^{2}=i d_{Y}$, but $f \neq i d_{Y}$.

(2) $f: Y \rightarrow Y$ is a rotation of period 3. Then $f$ is pointwise chain recurrent such that $f$ has exactly one fixed point.

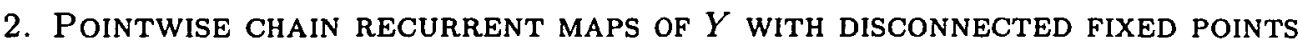 SET}

In this section, we assume that $f$ has a disconnected fixed points set. Then there exist two fixed points $a, b$ of $f$ with $(a, b) \cap F(f)=\phi$

THEOREM 2.1 If the closure of some component of $Y \backslash\{0\}$ contains $\{a, b\}$, then $f^{2}$ is turbulent. 
Proof: Without loss of generality, we assume that $\{a, b\} \subseteq\left[o, e_{1}\right]$ and $b<_{e_{1}} a$.

CASE 1. $f(x)<_{e_{1}} x$ for all $x \in(a, b)$. Then $b \neq e_{1}$, for otherwise $U=\left[e_{1}, a^{\prime}\right)$ satisfies $f(\bar{U}) \subseteq U$ for any $a^{\prime} \in(a, b)$. Let $c$ be the largest point in $\left(b, e_{1}\right]$ relative to $<_{e_{1}}$ such that $f(c)=a$. (If no such $c$ exists, then there exists $b^{\prime} \in(a, b)$ such that $f(x)<_{e_{1}} b^{\prime}$ for all $x \in\left(a, e_{1}\right]$. But then $U=\left(b^{\prime}, e_{1}\right]$ satisfies $f(\bar{U}) \subseteq U$.) Let $d \in(a, c)$ be the point with $f(d)=c$. (Again if no such $d$ exists, then there exists $c^{\prime} \in(b, c)$ such that $c^{\prime}<_{e_{1}} f(x)$ for all $x \in(a, c]$. But then $U=\left(d^{\prime}, c^{\prime}\right)$ satisfies $f(\bar{U}) \subseteq U$ for some $\left.d^{\prime} \in(a, b)\right)$. Then $J=[a, d]$ and $K=[d, c]$ show that $f$ is turbulent, and hence $f^{2}$ is turbulent.

CASE 2. $x<_{e_{1}} f(x)$ for all $x \in(a, b)$. There exists $c \in Y \backslash\left[a, e_{1}\right]$ such that $f(c)=b$, for otherwise, $U=Y \backslash\left[b^{\prime}, e_{1}\right]$ for some $b<_{e_{1}} b^{\prime}<_{e_{1}} a$ satisfies $f(\bar{U}) \subseteq U$. The following three subcases are considered.

SUBCASE 2.1. There exists $c_{i} \in\left[e_{i}, a\right]$ such that $f\left(c_{i}\right)=b, i=2,3$, and there exists $d_{2} \in\left[c_{2}, b\right]$ such that $f\left(d_{2}\right)=c_{2}$. (or there exists $c_{i} \in\left[e_{i}, a\right]$ such that $f\left(c_{i}\right)=b, i=2,3$, and there exists $d_{3} \in\left[c_{3}, b\right]$ such that $f\left(d_{3}\right)=c_{3}$, the proof of this case is similar and omitted.) Taking $J=\left[c_{2}, d_{2}\right]$ and $K=\left[d_{2}, b\right]$, one gets that $f(J) \cap f(K) \supseteq J \cup K$ and then $f$ is turbulent. Thus $f^{2}$ is turbulent.

SUBCASE 2.2. $b<_{e_{1}} f(x)$ for all $x \in\left[e_{3}, a\right]$ and there exists $c \in\left[e_{2}, o\right)$ such that $f(c)=b$. (or $b<_{e_{1}} f(x)$ for all $x \in\left[e_{2}, a\right]$ and there exists $c \in\left[e_{3}, o\right)$ such that $f(c)=b$, the proof of this case is similar and omitted.) Assume that such point $c$ is the largest one in $\left(e_{2}, 0\right)$ relative to $<_{e_{2}}$. Then there exists $d \in\left[e_{3}, b\right] \cup[c, o)$ such that $f(d)=c$. (If no such $d$ exists, then $U=\left[e_{3}, b^{\prime}\right) \cup\left(o, c^{\prime}\right)$ for some $b^{\prime} \in(a, b)$ and some $c^{\prime} \in(o, c)$ satisfies $f(\bar{U}) \subseteq U$.) If $d \in(c, b)$, then, taking $J=[c, d]$ and $K=[d, b]$, one gets that $f(J) \cap f(K) \supseteq J \cup K$ and thus $f^{2}$ is turbulent. Now, assume $[c, b] \cap f^{-1}(c)=\phi$ and such $d \in\left[e_{3}, o\right)$ is the largest one in $\left[e_{3}, o\right)$ relative to $<_{e_{3}}$. Then there exists $t \in[c, b] \cup[o, d]$ such that $f(t)=d$. (If no such $t$ exists, then $U=\left(c^{\prime}, b^{\prime}\right) \cap\left(d^{\prime}, o\right)$ for some $c^{\prime} \in(o, c)$, some $b^{\prime} \in(a, b)$ and some $d^{\prime} \in(o, d)$ satisfies $f(\bar{U}) \subseteq U$.) If $t \in(b, c)$, then, taking $J=[c, t], K=[t, b]$, one gets $f^{2}(J) \cap f^{2}(K) \supseteq J \cap K$ and thus $f^{2}$ is turbulent. If $t \in(o, d)$, then taking $J=[d, t], K=[t, b]$, one gets $f^{2}(J) \cap f^{2}(K) \supseteq[c, b] \cup[d, o] \supseteq J \cup K$ and thus $f^{2}$ is turbulent.

SUBCASE 2.3. $b<_{e_{1}} f(x)$ for all $x \in[o, a]$ and there exists $c_{i} \in\left[e_{i}, o\right)$ such that $f\left(c_{i}\right)=b$ and $f\left(\left[c_{i}, b\right]\right) \cap\left\{c_{i}\right\}=\phi, i=2,3$. Assume that such $c_{i}$ is the largest one in $\left[e_{i}, o\right)$ relative to $<_{e_{i}}, i=2,3$. Then there exists $d_{2} \in\left[c_{2}, o\right)$ such that $f\left(d_{2}\right)=c_{3}$ or $d_{3} \in\left[c_{3}, o\right)$ such that $f\left(d_{3}\right)=c_{2}$. (If none of such $d_{2}, d_{3}$ exists, then $U=\left(c_{2}^{\prime}, b^{\prime}\right) \cup\left(c_{3}^{\prime}, o\right)$ for some $c_{2}^{\prime} \in\left(c_{2}, o\right)$, some $c_{3}^{\prime} \in\left(c_{3}, o\right)$, and some $b^{\prime} \in(a, b)$ satisfies $f(\bar{U}) \subseteq U$.) Furthermore, assume that such $d_{i}$ is the largest one in $\left[c_{i}, o\right)$ relative to $<_{e_{i}}, i \in\{2,3\}$. Now a similar argument as that in Subcase 2.2 yields that $f^{2}$ is turbulent. The proof is complete.

THEOREM 2.2. If $a, b$ lie in two distinct components of $Y \backslash\{0\}, f^{2}$ is turbulent.

PROOF: Without loss of generality, assume that $b \in\left(o, e_{1}\right], a \in\left(o, e_{2}\right]$. 
CASE 1. $x<_{e_{1}} f(x)$ for all $x \in(o, b)$ (or $x<_{e_{2}} f(x)$ for all $x \in(o, a)$, the proof of this case is similar and omitted.) A similar proof as that of case 2 in Theorem 2.1 implies that $f^{2}$ is turbulent.

CASE 2. $f(x)<_{e_{1}} x$ for all $x \in(o, b)$ and $f(x)<_{e_{2}} x$ for all $x \in(o, a)$. Then $\left[a^{\prime}, b^{\prime}\right]$ $\cap F(f) \neq \phi$ for any $a^{\prime} \in(o, a)$ and any $b^{\prime} \in(o, b)$ (according to the proof of $[8$, Theorem 1 ], in fact, we have $o \in F(f))$. There is a contradiction. Therefore case 2 is impossible and proof is complete.

\section{Pointwise Chain RECURRENT MAPS of $Y$ WITH CONNECTED FIXED POINTS SET}

In this section, we assume that $f$ has connected fixed points set. Then $F(f)$ is a connected closed subset of $Y$. If $F(f)$ is degenerated, then $f$ has exactly one fixed point. This case will be discussed in section 4. Now assume that $F(f)$ is nondegenerated.

THEOREM 3.1 If $F(f)$ is contained in the closure of a component of $Y \backslash\{0\}$, then $f^{2}=i d_{Y}$ but $f \neq i d_{Y}$ or $f^{2}$ is turbulent.

Proof: Without loss of generality, assume that $F(f)=[p, q] \subseteq\left[o, e_{1}\right]$ and $p<_{e_{1}} q$.

We first claim that $q=o$. Suppose not. Then $f(x)<_{e_{1}} x$ for all $x \in[o, q]$. Note that $p, q$ are fixed points of $f$. There exists $q^{\prime} \in(o, q)$ such that $f\left(\left[q^{\prime}, p^{\prime}\right]\right) \subseteq\left(q^{\prime}, p^{\prime}\right)$ for some $p^{\prime} \in\left(p, e_{1}\right)$ (if $\left.p \neq e_{1}\right)$ or $f\left(\left[q^{\prime}, e_{1}\right]\right) \subseteq\left(q^{\prime}, e_{1}\right]$ (if $p=e_{1}$ ). There is a contradiction. By the claim, the following two cases will be considered.

CASE 1. $p \neq e_{1}$. Clearly, we have $x<_{e_{1}} f(x)$ for all $x \in\left(p, e_{1}\right] ; x<_{e_{2}} f(x)$ for all $x \in\left(o, e_{2}\right]$ and $x<_{e_{3}} f(x)$ for all $x \in\left(o, e_{3}\right)$. Since $f$ is onto, there exists $x_{0} \in\left[e_{2}, e_{3}\right] \backslash\{o\}$ such that $f\left(x_{0}\right)=e_{1}$. Without loss of generality, we assume that $x_{0} \in\left[e_{2}, o\right)$. Then, by the continuity of $f$, there exists $r \in\left(o, x_{0}\right)$ such that $f(r)=p$. Furthermore, we may assume that such $r$ is the largest one in $\left(e_{2}, o\right)$ relative to $<_{e_{2}}$.

SubCASE 1.1. $p<_{e_{1}} f(x)$ for all $x \in\left(o, e_{3}\right]$. Then there exists $s \in(o, r) \cup\left(o, e_{3}\right]$ such that $f(s)=r$. (If no such $s$ exists, then $U=\left(r^{\prime}, e_{3}\right] \cup\left(o, p^{\prime}\right)$ for some $r^{\prime} \in(o, r)$ and some $p^{\prime} \in\left(p, e_{1}\right)$ satisfies $\left.f(\bar{U}) \subseteq U.\right)$ Furthermore, we have $s \in\left(o, e_{3}\right.$ ] (for otherwise $(o, r) \cap F(f) \neq \phi)$ and assume that such $s$ is the largest one in $\left(o, e_{3}\right]$ relative to $<_{e_{3}}$. There exists $t \in(o, r) \cup(o, s)$ such that $f(t)=s$. (If no such $t$ exists, then $U=\left(r^{\prime}, s^{\prime}\right) \cup\left(o, p^{\prime}\right)$ for some $r^{\prime} \in(o, r)$, some $s^{\prime} \in(o, s)$ and some $p^{\prime} \in\left(p, e_{1}\right)$ satisfies $f(\bar{U}) \subseteq U$.) Furthermore, we have $t \in(o, r)$ (for otherwise, $(o, s) \cap F(f) \neq \phi$ ). Taking $J=[o, t], K=[t, r]$, one gets $f^{2}(J) \cap f^{2}(K) \supseteq J \cup K$ and thus $f^{2}$ is turbulent.

SUBCASE 1.2 There exists $r_{1} \in\left(o, e_{3}\right)$ such that $f\left(r_{1}\right)=p$. Without loss of generality, assume that such $r_{1}$ is the largest one in $\left[e_{3}, o\right)$ relative to $<_{e_{3}}$. Then there exists $s \in\left(o, r_{1}\right)$ such that $f(s)=r$ or $s_{1} \in(o, r)$ such that $f\left(s_{1}\right)=r_{1}$. (If none of such $s, s_{1}$ exists, then $U=\left(r^{\prime}, r_{1}^{\prime}\right) \cup\left(o, p^{\prime}\right)$ for some $r^{\prime} \in(o, r), r_{1}^{\prime} \in\left(o, r_{1}\right)$ and some $p^{\prime} \in\left(p, e_{1}\right]$ satisfies $f(\bar{U}) \subseteq U$.$) Without loss of generality, we assume that there exists s \in\left(o, r_{1}\right)$ such 
$f(s)=r$. (If there exists $s_{1} \in(o, r)$ such that $f\left(s_{1}\right)=r_{1}$, the proof of this case is similar and omitted.) A similar argument as that in subcase 1.1 yields that $f^{2}$ is turbulent.

CASE 2. $p=e_{1}$. Clearly, we have $x<_{e_{2}} f(x)$ for all $x \in\left(o, e_{2}\right]$ and $x<_{e_{3}} f(x)$ for all $x \in\left(o, e_{3}\right]$.

If there exists $a \in\left[e_{2}, e_{3}\right] \backslash\{o\}$ such that $f(a) \in\left(o, e_{1}\right]$, then we can get $b \in(o, a)$ $\cup\left(o, e_{3}\right]$ (without loss of generality, assume that $a \in\left(o, e_{2}\right]$. For $a \in\left(o, e_{3}\right]$, a similar argument will be done.) such that $f(b)=a$. (If no such $b$ exists, then there exists $a^{\prime} \in(o, a)$ such that $a^{\prime}<_{e_{2}} f(x)$ for all $x \in(o, a] \cup\left(o, e_{3}\right]$. But then $U=\left[e_{1}, e_{3}\right] \cup\left(o, a^{\prime}\right)$ satisfies $f(\bar{U}) \subseteq U)$.) In fact, we have $b \in\left(o, e_{3}\right]$. (For otherwise, $F(f) \cap(o, a) \neq \phi$.) Without loss of generality, assume that such $b$ is the largest one in $\left(o, e_{3}\right]$ relative to $<_{e_{3}}$ such that $f(b)=a$. Furthermore, let $c$ be any point in $(a, b)$ such that $f(c)=b$. (Again if no such $c$ exists, then there exists $b^{\prime} \in(o, b)$ such that $b^{\prime}<_{e_{3}} f(x)$ for all $x \in[a, b] \cup\left(o, e_{1}\right]$. But then $U=\left(a^{\prime}, b^{\prime}\right) \cup\left(o, e_{1}\right]$ satisfies $f(\bar{U}) \subseteq U$ for some $a^{\prime} \in(o, a)$.) In fact, we have $c \in(o, a)$ (for otherwise, $\left.F(f) \cap\left(o, e_{3}\right] \neq \phi\right)$ Taking $J=[o, c], K=[a, c]$, one gets $f^{2}(J) \cap f^{2}(K) \supseteq J \cup K$ and thus $f^{2}$ is turbulent.

If $f^{-1}\left(\left(o, e_{1}\right]\right) \cap\left[e_{2}, e_{3}\right]=\phi$, then $\left.f\right|_{\left[e_{2}, e_{3}\right]}:\left[e_{2}, e_{3}\right] \rightarrow\left[e_{2}, e_{3}\right]$ is pointwise chain recurrent and has exactly one fixed point. It follows from $\left[4\right.$, Theorem] that $\left.f^{2}\right|_{\left[e_{2}, e_{3}\right]}$ $=\left.i d\right|_{\left[e_{2}, e_{3}\right]}$ or $\left.f^{2}\right|_{\left\{e_{2}, e_{3}\right]}$ is turbulent. If $\left.f^{2}\right|_{\left\{e_{2}, e_{3}\right]}=\left.i d\right|_{\left[e_{2}, e_{3}\right]}$ then $f^{2}=i d_{Y}$ but $f \neq i d_{Y} ;$ if $\left.f^{2}\right|_{\left[e_{2}, e_{3}\right]}$ is turbulent, then $f^{2}$ is certainly turbulent.

The proof is complete.

THEOREM 3.2. There does not exist $f$ such that $o \in \operatorname{int} F(f)$ except the identity map $i d_{Y}$.

Proof:Assume that such $f$ exists and $f$ is not the identity. Let $F(f) \cap\left[0, e_{i}\right]$ $=\left[o, p_{i}\right], i \in\{1,2,3\}$. Note that each $p_{i}$ is the smallest fixed point in $\left[0, e_{i}\right]$ relative to $<_{e_{i}}$. Then there exists $p_{i}^{\prime} \in\left(p_{i}, e_{i}\right)$ (if $\left.p_{i} \neq e_{i}\right)$ such that $x<_{e_{i}} f(x)<_{e_{i}} p_{j}(i \in\{1,2,3\}$, and $j \neq i$ ) for all $x \in\left(p_{i}, p_{i}^{\prime}\right)$. Thus, taking

$$
U=U_{1} \cup U_{2} \cup U_{3},
$$

where each $U_{i}=\left[o, p_{i}^{\prime}\right)$ if $p_{i} \neq e_{i} ;\left[o, e_{i}\right]$ if $p_{i}=e_{i}$, one gets that $f(\bar{U}) \subseteq U$. There is a contradiction. The proof is complete.

\section{Pointwise Chain RECURRENT of $Y$ With EXACT ONE FIXED POINT}

In this section, we assume that $f$ has exactly one fixed point, written by $p$.

LEMMA 4.1.

(1) If $p=o$, then $f^{2}$ has exactly one fixed point too, but then $f^{3}$ has more than one fixed point.

(2) If $p \neq o$, then $f^{2}$ has more than one fixed point. 
Proof: (1) Assume that $f^{2}$ has a fixed point $p^{\prime}$ different from $o$. Without loss of generality, we assume that $p^{\prime} \in\left(o, e_{1}\right]$, then $f\left(p^{\prime}\right) \in\left(o, e_{3}\right] \cup\left(o, e_{2}\right]$ (for otherwise, there exists at least one fixed point of $f$ in $\left(o, e_{1}\right]$.) Without loss generality, we assume that $f\left(p^{\prime}\right) \in\left(o, e_{2}\right]$. Since $f$ is onto, there exist $a_{1} \in\left(o, e_{2}\right] \cup\left(o, e_{3}\right]$ such that $f\left(a_{1}\right)=e_{1}$, $a_{2} \in\left(o, e_{1}\right] \cup\left(o, e_{3}\right]$ such that $f\left(a_{2}\right)=e_{2}$ and $a_{3} \in\left(o, e_{1}\right] \cup\left(o, e_{2}\right]$ such that $f\left(a_{3}\right)=e_{3}$. If $a_{1} \in\left(o, e_{2}\right]$, then we claim that $a_{2} \in\left(o, e_{3}\right)$ and $a_{3} \in\left(o, e_{1}\right]$ (If $a_{1} \in\left(o, e_{3}\right]$, we must have $a_{2} \in\left(o, e_{1}\right]$ and $a_{3} \in\left(o, e_{2}\right]$. A similar argument will be done.) In fact, if $a_{2} \in\left(o, e_{1}\right]$, then $a_{3} \in\left(o, e_{2}\right]$ or $a_{3} \in\left(o, e_{1}\right]$. Without loss of generality, we assume that $a_{3} \in\left(o, e_{2}\right]$ (If $a_{3} \in\left(o, e_{1}\right]$, the proof of this case is similar and omitted.) Furthermore, we assume that $a_{1}<_{e_{2}} a_{3}$ (If $a_{3}<_{e_{2}} a_{1}$, the proof of this case is similar and omitted.), then by the continuity of $f, f\left(a_{3}\right) \in\left[o, e_{1}\right]$, which contradicts $f\left(a_{3}\right)=e_{3}$. Thus, we have $p^{\prime}, a_{3} \in\left(o, e_{1}\right]$. By the continuity of $f$, if $p^{\prime}<_{e_{1}} a_{3}$, then $f\left(a_{3}\right) \in\left[o, f\left(p^{\prime}\right)\right]$, which contradicts $f\left(a_{3}\right)=e_{3}$; If $a_{3}<_{e_{1}} p^{\prime}$, then $f\left(p^{\prime}\right) \in\left[o, e_{3}\right]$, which contradict $f\left(p^{\prime}\right) \in\left(o, e_{2}\right]$.

From the above discussion, we see that either there exist $a_{1} \in\left(o, e_{2}\right], a_{2}$ $\in\left(o, e_{3}\right], a_{3} \in\left(o, e_{1}\right]$, or $a_{1} \in\left(o, e_{3}\right], a_{2} \in\left(o, e_{1}\right], a_{3} \in\left(o, e_{2}\right]$ such that $f\left(a_{1}\right)=e_{1}, f\left(a_{2}\right)$ $=e_{2}, f\left(a_{3}\right)=e_{3}$. Since the proofs of the above two cases are similar. We only prove the former. Clearly, $\left[o, a_{1}\right] \subseteq f^{3}\left(\left[o, a_{1}\right]\right)$, hence there exists $a \in\left[o, a_{1}\right]$ such that $f^{3}(a)=a_{1}$. Then $f^{3}$ has a fixed point in $\left[a, e_{2}\right]$.

(2) In fact, if $p \neq o$, then we must have $p$ is in one component of $Y \backslash\{o\}$ and $p \notin\left\{e_{1}, e_{2}, e_{3}\right\}$ (For otherwise, there exist more than one fixed point of $f$.). The proof of this case is similar to that of [4, Lemma 3$]$ and omitted.

THEOREM 4.1.

(1) If $p=o$, then $f^{2}$ can not be turbulent. But $f^{6}$ is turbulent or identity map.

(2) If $p \neq o$, then $f^{4}$ is turbulent or identity map.

Proof: By the previous results, the theorem is clear. Now to prove the main theorem, by Theorems 2.1, 2.2, 3.1, 3.2 and Lemma 4.1 , either $f^{12}$ is the identity map or $f^{12}$ is turbulent.

\section{REFERENCES}

[1] M. Barge and J. Martin, 'Dense periodicity on the interval', Proc. Amer. Math. Soc. 94 (1985), 731-735.

[2] L. Block and W.A. Coppel, 'Stratification of continuous maps of an interval', Trans. Amer. Math. Soc. 297 (1986), 587-604.

[3] L. Block and W.A. Coppel, Dynamics in one dimension, Lecture Notes in Math. 1513 (Springer-Verlag, Berlin, Heidelberg, New York, 1991).

[4] L. Block and E.M. Coven, 'Maps of the interval with every point chain recurrent', Proc. Amer. Math. Soc. 98 (1986), 513-515.

[5] L. Block and J. Franke, 'The chain recurrent set, attractors, and explosions', Ergodic Theory Dynamical Systems 5 (1985), 321-327. 
[6] T. Li and X.D. Ye, 'Chain recurrent point of a tree map', Bull. Austral. Math. Soc. 59 (1999), 181-186.

[7] S.B. Nadler, Jr., Continum theory. An introduction (Marcel Dekker, Inc., New York, Basel. Hong Kong, 1992).

[8] F.P. Zeng, H. Mo and W.J. Guo, ' $\omega$-limit points of a tree map', in International Conference on Foundations of Computational Mathematics in Honor of Professor Steve Smale's 70th Birthday, July 13-17 (City University, Hong Kong, 2000).

[9] J. Zhang and J. Xiong, Interations of function and one-dimension dynamics, (in Chinese) (Sichuan Education Press,, 1992).

School of Economics Management

Xidian University

Xi'an

Shanxi 710071

People's Republic of China

School of Economics Management

Xidian University

Xi'an

Shanxi 710071

People's Republic of China
Institute of Mathematics

Guangxi University

Nanning

Gangxi 530004

People's Republic of China 